\title{
High Resolution DEMs from Unmanned Aerial Vehicles
}

\author{
$\underline{\text { Trevor I Dowling }}{ }^{\text {a }}$ and John C Gallant ${ }^{\text {a }}$ \\ ${ }^{a}$ CSIRO, Land and Water, Canberra \\ Email: john.gallant@csiro.au
}

\begin{abstract}
Demand for high resolution DEMs in Australia seems to be insatiable. Released in 2012, the national 1 second resolution (30m) DEMs derived from SRTM provided a consistent high resolution continental coverage, though there are always applications that require better resolution. This is particularly the case where river channels that are less than one grid cell in width are involved. Examples where channel shape is important include hydrodynamic modeling and dam siting.
\end{abstract}

Previous papers by the authors at Modsim07 and Modsim09 described the need for more frequent survey with finer DEM resolution and the potential for cost effective methods such as Photosynth, which has since been joined by a number of commercial and open source competitors. A major obstacle in that method was the transformation from model to world coordinates. Recent developments in this area include the ability to work directly in world coordinates using air photos tagged with GPS camera position coordinates. Alongside software development has been the rapid development of low-cost and effective Unmanned Aerial Vehicles (UAVs) and cameras capable of obtaining high quality aerial photographs and video. The main advantages of these platforms are their relative low cost, less dependence on contractor schedules, and the flexibility to select the conditions and fly as frequently as necessary to achieve a desired result.

This paper describes the methods used in setting up a UAV and obtaining air photos, together with the use of freely available Structure from Motion (SFM) software to derive a high resolution DEM to supplement the national 1" DEM. Comparisons of DEMs with the 1" DEM show the effectiveness of relatively modest inputs.

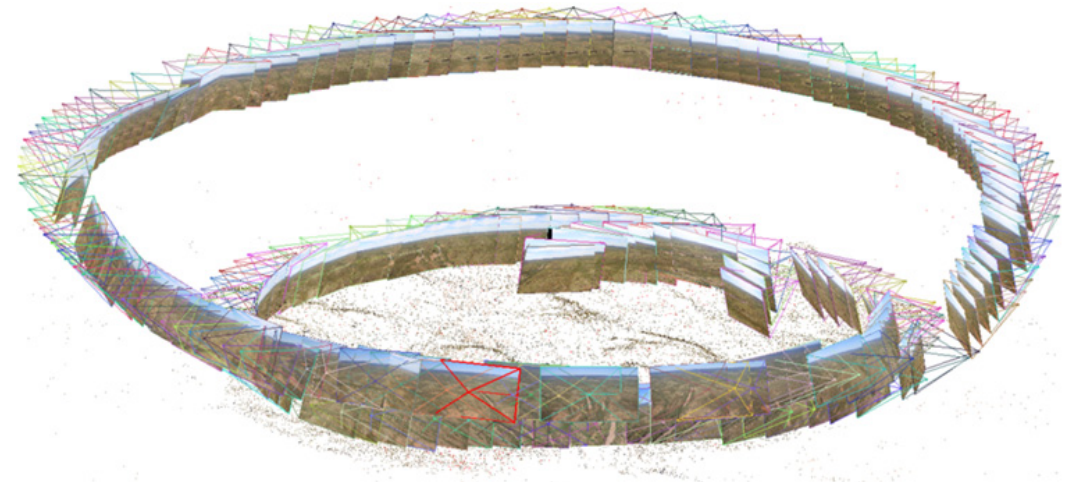

Figure 1. Airphoto camera positions and the derived point cloud which is subsequently interpolated at $2.5 \mathrm{~m}$ resolution.
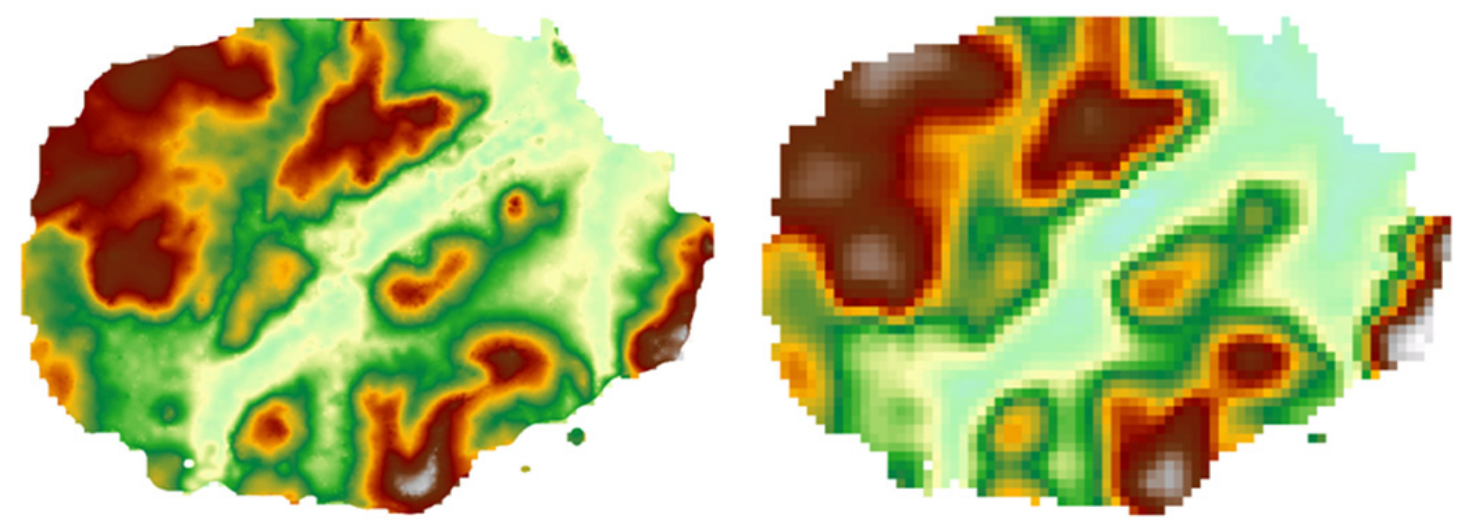

Figure 2. Comparison of $2.5 \mathrm{~m}$ resolution Structure from Motion (left) and 1 second (30m) (right) DEMs

Keywords: DEM, Structure from motion, Unmanned Aerial Vehicle 


\section{INTRODUCTION}

Demand for high resolution DEMs in Australia seems to be insatiable. Released in 2012, the national 1 second resolution (30m) DEMs derived from SRTM (Gallant et al., 2011), provided a consistent high resolution continental coverage. Despite being a significant improvement over the existing 9 second DEM (GA, 2008) there are always applications that require better resolution. This is particularly the case for the representation of river channels that are less than one grid cell in width. Examples where channel shape is important include hydrodynamic modeling and dam siting.

Previous papers by the authors (Dowling et al., 2007 and Dowling et al., 2009) described the need for more frequent survey with finer DEM resolution and the potential for cost effective methods such as Photosynth (an online multi-angle photogrammetry solution) which has since seen competition from a number of commercial and open source alternatives. A major obstacle when using Photosynth was establishing control points for the transformation from model to real-world coordinates, and recent developments in alternative software includes the ability to work directly in real-world coordinates using air photos tagged with GPS camera position coordinates.

Alongside software development has been the rapid development of low-cost and effective Unmanned Aerial Vehicles (UAVs) and very compact cameras capable of obtaining high quality aerial photos and video. The main advantages of these platforms are relative low cost, independence from survey contractor schedules, and the flexibility to pick the conditions and fly as frequently as necessary to achieve a desired result.

Currently work is focused on investigating flight planning to optimize the flying time, the survey coverage and the quality of imagery (best orientation and overlaps) to suit Structure from Motion (SFM) applications. This paper describes the methods used in setting up a UAV and obtaining air photos, together with the use of freely available SFM software to derive high resolution DEMs to supplement the national 1" DEM.

\section{METHODS}

\subsection{Structure from Motion (SFM) software}

While there several open source and commercial Structure from Motion (SFM) applications available the one chosen for this work to derive Digital Surface Models (DSMs) is called Visual SFM (Changchang, 2012; 2013). Previously Dowling et al. (2009) used Photosynth (Microsoft, 2009) to derive a high quality $2.5 \mathrm{~cm}$ resolution DEM of a 1 x $1 \mathrm{~m}$ erosion site. The difficulty with this method was identifying unambiguous real world coordinates in the noisy point cloud produced by Photsynth in order to perform a coordinate transformation. Visual SFM (VSFM) has the advantage that it can incorporate GPS coordinates of camera positions and has the transformation functionality included.

The requirement of SFM is a set of overlapping images with sufficient resolution to match patterns in images at the required level of detail. The quality of the model is dependent on resection, where points viewed from at least three photographs with orthogonal (as wide as possible) directions and heights is best. Picture quality is less critical and frames extracted from HD video as .jpg images were found to be adequate.

Processing image matches and resections is very intensive and was performed on a dual processor Xeon E5506 $2.13 \mathrm{GHz}$ CPUs with 25GB RAM and GPU. Processing for the largest dataset (840 images) took several hours.

\subsection{Data acquisition}

Many camera platforms and cameras and GPSs are now available at varying, though generally decreasing, cost and choice of equipment requires careful consideration. Current testing has involved comparing several types of platforms and 5 HD video cameras. The scenarios covered here are:

- Handheld digital HD video camera and a handheld GPS in a helicopter.

- "Keyring" HD 720 and 1080 cameras mounted on a small UAV capable of autonomously flying preprogrammed missions, which is the main focus of this research.

The handheld video from helicopter was collected as part of a familiarization survey where the helicopter was flown slowly in a descending spiral with a good quality video camera centred on the site of interest, while a GPS collected position information. (Figure 1). The video was converted to 1 second interval images using a bespoke Python script. The 200 images were matched with the GPS log file to obtain a camera positions text file containing image-name, longitude, latitude and elevation that was used by VSFM. The point cloud from the best VSFM model was exported, projected from earth geocentric coordinates to WGS84 
and written as a shapefile in a python script. The shapefile points were detrended by calculating differences between spot heights and 1" DEM-S, deriving a first-order difference surface and adjusting spot heights accordingly. Adjusted spot heights were interpolated in ArcMap using the inverse distance weighted (IDW) tool into a $2.5 \mathrm{~m}$ resolution DSM covering an area of approximately $2 \times 1.6 \mathrm{~km}$.

For the UAV-mounted "Keyring" acquisition, a small area was flown in a mission that entailed three circles around the 'target' at varying radius and elevation. The images relating to each circle at $40 \mathrm{~m}, 80 \mathrm{~m}$ and $120 \mathrm{~m}$ elevation, approximately $140,210,300 \mathrm{~m}$ diameter respectively, were manually extracted from the dataset to assess the relative contribution of each as well as using all three combined. When initial results were less than impressive the images were re-extracted at 0.5 second interval and some additional untargeted video captured before and after the circles on the same flight was incorporated. Post-processing was similar to the helicopter dataset with the exception that the interpolation was performed using ArcMap Topogrid without the drainage enforcement option.

Regardless of the platform from which the video or still images are obtained the flight path needs to be optimized to:

- maximize the area covered in a mission (i.e. flying time which is a function of cost, battery life, memory card capacity etc)

- reduce the amount of redundant processing in VSFM which is already very computationally intensive

- $\quad$ fill a niche between large high cost conventional aircraft that are more cost effective for large areas and the low cost multi rotor UAVs which have a relatively limited range.

\subsection{Unmanned Aerial Vehicles}

"Turnkey" UAV systems with all components integrated are commercially available, for example the Gatewing X100 (http://uas.trimble.com), but these remain quite expensive for irregular use on small projects. On the other hand, building a UAV from parts is relatively easy and cheap. The technology, software and advice for building fully stabilized and autonomous systems are readily available from special interest groups (DIYDrones, 2013; CUAV, 2013). The platform used for this work (Figure 3) was built from parts for less than AUD\$1800. It uses Arduplane autopilot and a laptop running MissionPlanner (DIY Drones, 2013).

In either case a responsible entity is required to maintain, fly and control a UAV system. There are increasing public concerns about safety and privacy leading to more regulation and responsibility and consequences for accidents or breaches of air safety and privacy laws can be severe. The rules around the use of UAVs for private versus commercial use are arbitrary and alone may preclude the use of a UAV for many purposes or require significant expense in training and maintenance. Currently the Civil Aviation Safety Authority (CASA) is developing new rules which include variations for various weight classes and guidelines are imminent.

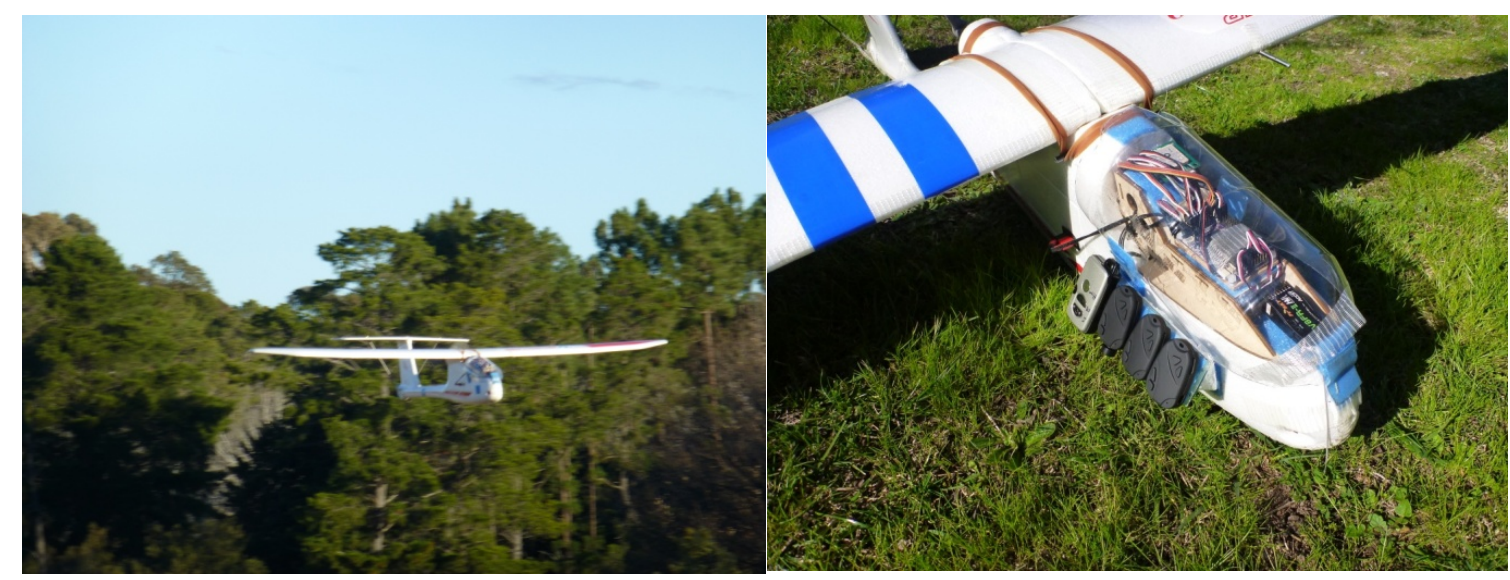

Figure 3. The UAV platform used in this study and a close up showing the cameras being tested and the electronics required for manual and/or autonomous flight. The current configuration has a $1.9 \mathrm{~m}$ wingspan and weighs approximately $2.5 \mathrm{~kg}$. 
The main considerations in choosing this platform were:

- Cost - had to be affordable for modest projects and replaceable in the event of (inevitable) crashes. Cost of enough spare parts, or optimally a spare UAV is important.

- Portability - able to transport to remote locations and set up easily. Hand launch requires minimal distance and belly landings require less than one hectare of clear space. Electric motor with lithium polymer (LiPo) batteries was chosen for cleanliness and vibration reduction although fire risk and transport (crucially for commercial air transport) is as much a concern as liquid fuels.

- Endurance - using a 5Ah 4 cell LiPo battery flying time at a cruise speed of $\sim 30 \mathrm{~km} / \mathrm{h}$ is $\sim 50$ minutes, a distance of $\sim 25 \mathrm{~km}$.

- $\quad$ Payload - enough capacity for multiple cameras and longer range batteries if desired.

- $\quad$ Stability - easy to fly, stable, minimal vibration, ability to preprogram flights helps smooth flight control and autonomous missions out of visual range. Stabilised flight helps hand launching and belly landing.

- $\quad$ Portability - able to transport to remote locations and set up easily. Hand launch requires minimal distance and belly landings require less than one hectare of clear space. Electric motor with lithium polymer ( $\mathrm{LiPo}$ ) batteries was chosen for cleanliness and vibration reduction although fire risk and transport (crucially for commercial air transport) is as much a concern as liquid fuels.

- Robustness - a pusher propeller above the fuselage and foam construction make for a system resistant to damage and readily repairable when damage does occur.

\section{RESULTS}

\subsection{Helicopter dataset}

Conversion of the video resulted in 200 one second interval images which produced a point cloud of 56,184 points in VSFM (Figure 1). After post processing and interpolation the resulting DSM with 2.5m resolution covered an area of $2000 \times 1600 \mathrm{~m}$ (Fig. 2). Visual inspection of the DSMs with identical color scales (Figure 2) and the difference map (Figure 4b) indicates that there is a good correlation between overall elevations with a marked improvement in detail. Most of the difference occurs along the river banks and on hilltops.

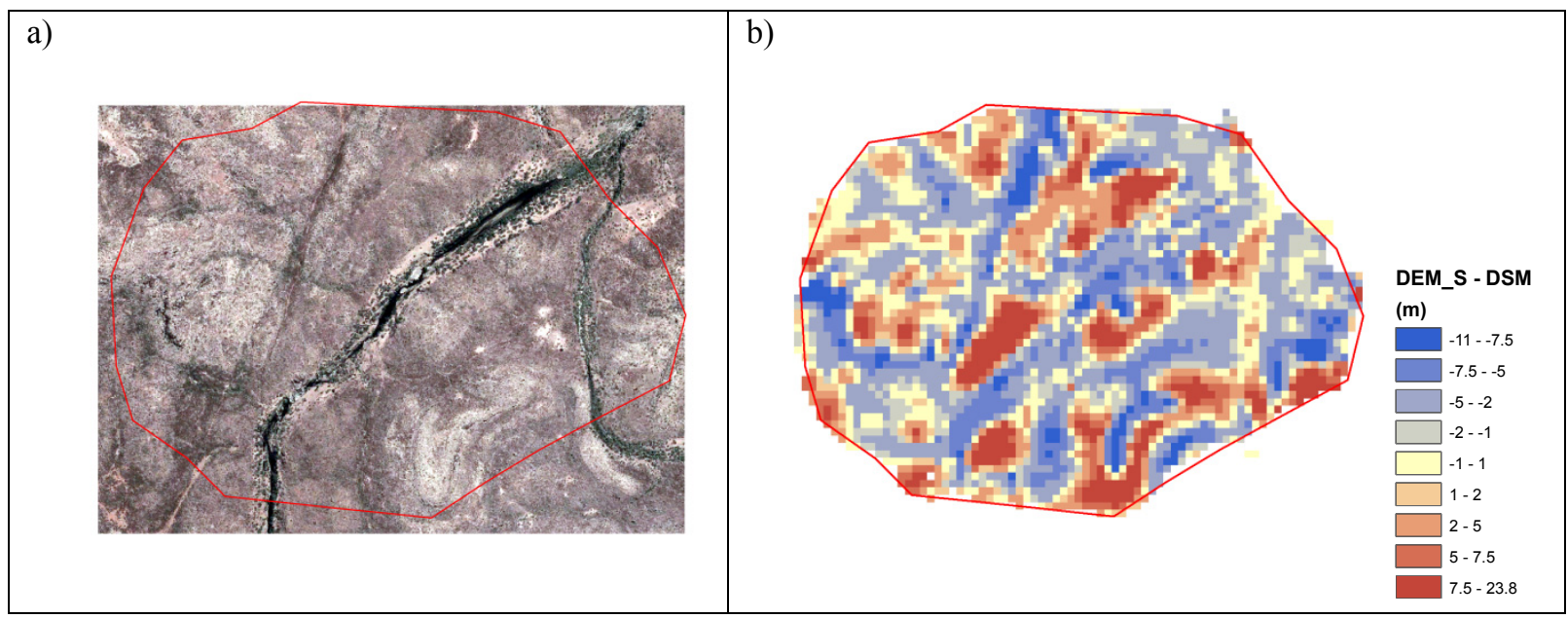

Figure 4. a) Google Earth image of the 'Helicopter' site and b) the difference between 1" (30m) DEM-S and VSFM 2.5m DEM (see Figure 2). Fine-scale differences along the river bed demonstrate differences in resolving power of channels and the effect of trees, and the larger red areas are small hills that are not fully represented in the coarser DEM-S.

\subsection{UAV dataset}

The 40m altitude images, 28 in total, produced an unusable sparse point cloud in VSFM (Fig. 5a) and covered very little of the target area as anticipated. It produced many partial models indicating a lack of overlap and geometry in the images. 
The $80 \mathrm{~m}$ altitude circle, consisting of 44 images improved the density of the point cloud (Fig. $5 \mathrm{~b}$ ) but still produced numerous partial models.

The $120 \mathrm{~m}$ circle, consisting of 64 images, was a further improvement, though still produced 6 models of variable quality (Fig. 5c). This resulted in 32,094 points in the cloud of the best model.

All data at 1 second interval resulted in 423 images, 113,886 points from VSFM (Fig. 5d) and a $5 \mathrm{~m}$ resolution DSM covering $300 \times 350 \mathrm{~m}$.

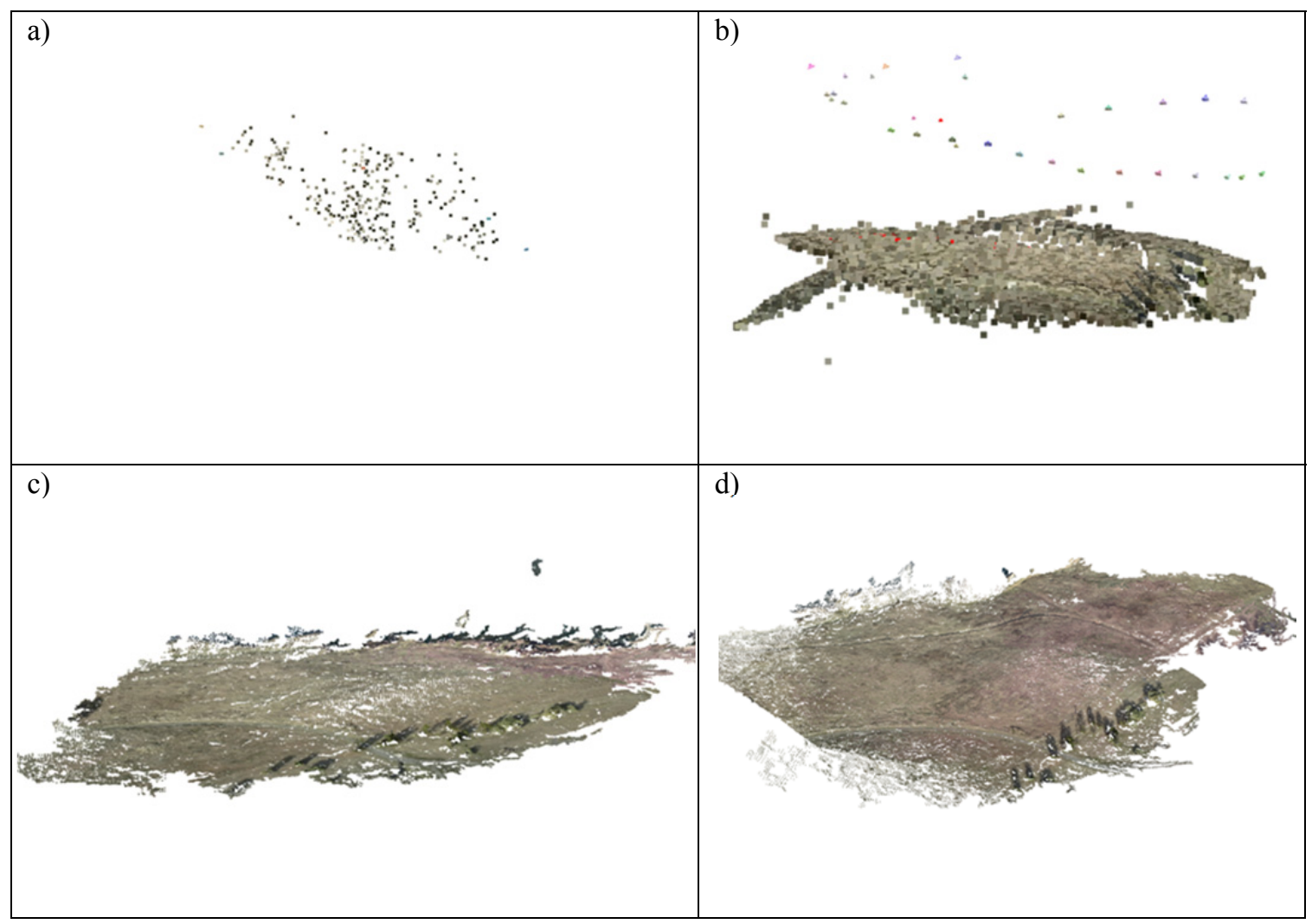

Figure 5. Point clouds derived using imagery from a wide angle 'keyring' video recorder mounted on the UAV at a) $40 \mathrm{~m}$ altitude, b) $80 \mathrm{~m}$, c) $120 \mathrm{~m}$ and d) all useable images.

None of the above DSMs were considered worth further analysis and a final investigation of the UAV dataset involved re-extracting images at 0.5 second interval and using all available images from the flight resulting in 845 images in the combined dataset and 119,252 points in the VSFM point cloud. The DSM derived from the point cloud was interpolated at $5 \mathrm{~m}$ resolution and covers an area of $300 \times 350 \mathrm{~m}$ (Fig. 5d). The difference between the 1" DEM-S and the $5 \mathrm{~m}$ DSM derived from the point cloud, obtained using all photos, is shown in Fig. 6d.

After de-trending the UAV spot heights a second DSM was interpolated and the difference from the DEM-S calculated as illustrated in Fig. 6e, f. The central area of the VSFM DSM in Figure 6e (within the roughly circular flight paths) corresponds well with visual observations of the site and is a much better representation of the site topography than the 1" DSM. The DSM does contain some point-like artefacts (both high and low) that would require treatment before the DSM was ready for use. 


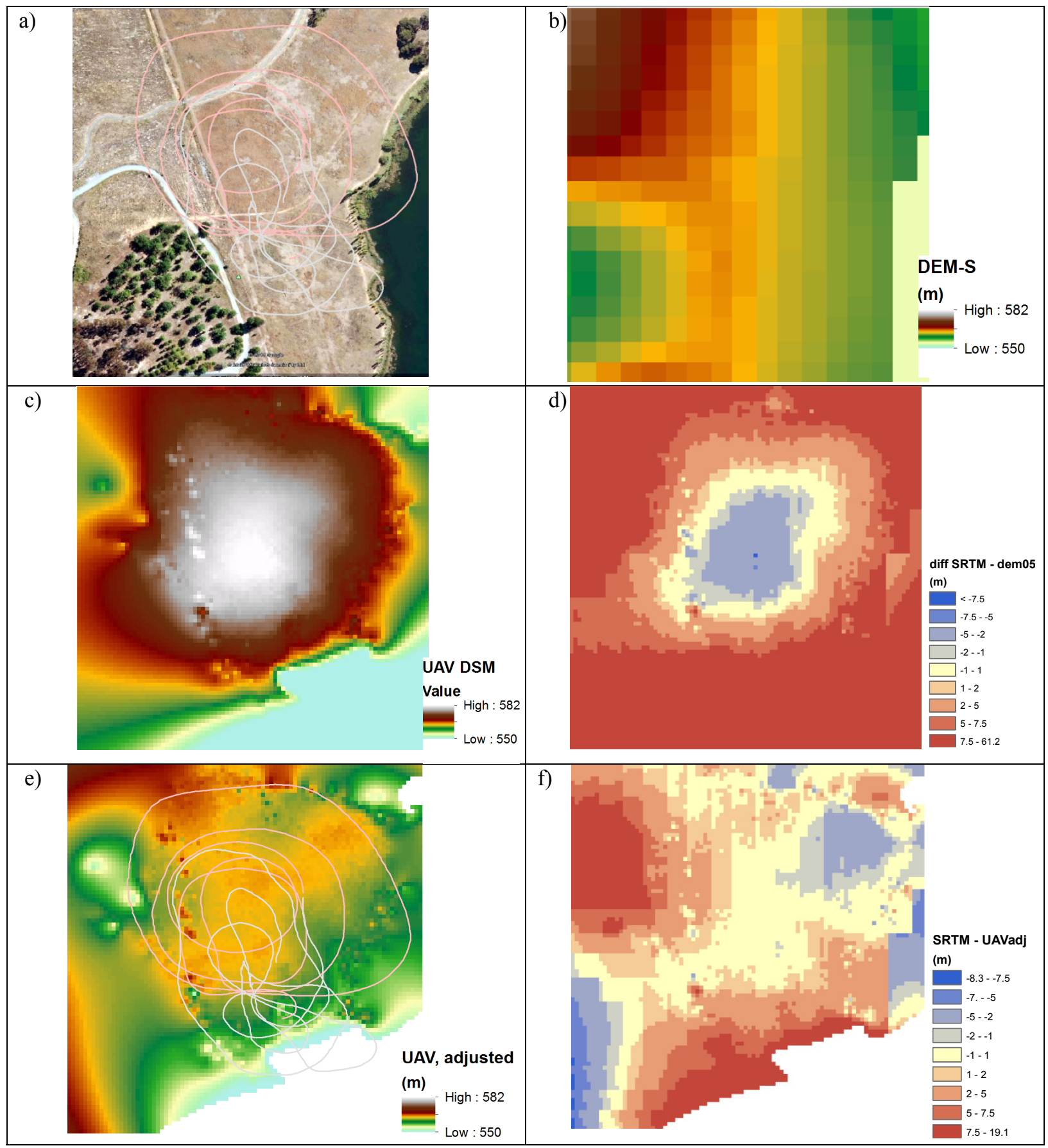

Figure 6. a) Study site, b) the 1" DEM-S, c) VSFM 5m DEM, d) the difference (b minus c), e) The VSFM $5 \mathrm{~m}$ DSM adjusted for wide angle lens induced distortion and $\mathrm{f}$ ) the difference (b minus e).

\section{DISCUSSION}

Use of helicopters and other aircraft, particularly for small projects, can be prohibitive e.g. generally expensive, restrictive schedules, increased administration and lack of repeatability (more necessary for higher resolution DEMs). Regardless of the platform or cameras there are many considerations for obtaining optimal air photos for use in VSFM including: what is the best flight path, camera, image obliqueness, focal length, altitude/distance from target and proximity of overlapping images.

Considering the simplicity of the flight path, the helicopter dataset produced an adequate fit-for-purpose DSM, adding substantial detail to the $\sim 30 \mathrm{~m}$ DEM-S for relatively little effort. This survey used a higher quality video camera which appears to avoid the curvature (dome) distortion problem illustrated in Fig. 6c,d. 
This occurred because the camera focal length was not known and the method VSFM uses to estimate it was inadequate. While detrending seems to produce reasonable results it is best to supply actual focal lengths to VSFM if known. The advantage of the wide angle camera is that it is easier to aim and increases the area that can be surveyed.

Some of the differences between DEM-S and VSFM are due to trees which will be 'seen' differently by radar and SFM. Tree offsets in the SRTM data were removed during processing from DSM to DEM-S, but tree cover in the area also changed between the SRTM acquisition (2000) and the UAV acquisition (2013).

\section{CONCLUSIONS}

The SFM software tools have matured greatly since our initial attempts with Photosynth. The ability to input camera positions and transform point cloud coordinates has improved the workflow considerably.

UAVs can fly a preprogrammed set of waypoints but the choice of an efficient flight plan to obtain the highest quality model from SFM is important and the focus of current testing. Future testing is anticipated that will incorporate the ability for the autopilot to trigger high quality still cameras at waypoints and to control a gimbal mount to improve camera aiming.

The equipment and software are relatively inexpensive and becoming more so every day. Together with other advantages, such as portability, flexibility for opportunistic flying in optimal conditions and repeatability this technology can easily be added into other field work at relatively low cost.

While being a useful and exciting technology UAVs may not be for general use because of the significant air safety, maintenance, privacy and legal implications.

\section{ACKNOWLEDGMENTS}

The UAV was funded by the TERN Auscover project to enhance the capability for land cover measurement using alternative sensors as they become ever smaller.

\section{REFERENCES}

CUAV (2013) Canberra Unmanned Aerial Vehicles Website: http://www.canberrauav.com, July 2013.

Cangchang, Wu 2(012) VisualSFM : A Visual Structure from Motion System. Univ. of Washington, Seattle. http://homes.cs.washington.edu/ ccwu/vsfm/

Changchang, Wu (2013) "Towards Linear-time Incremental Structure from Motion", 3DV 2013 3DV Conference (Third Joint 3DIM/3DPVT Conference), Seattle, Washington, Jun 29, 2013 - Jul 1, 2013. http://homes.cs.washington.edu/ ccwu/vsfm/vsfm.pdf

DIY Drones, Arduplane, Mission planner. http://diydrones.com/ July 2013

Dowling, T.I, Richardson, D.P. and Gallant, J.C. (2007), How high is high resolution? Implications of a veryhigh-resolution micro-relief erosion study. Proceedings MODSIM07, 9-13 Dec 2007, Christchurch, NZ, Christchurch, NZ, 9-13 Dec 2007, Conference CD.

Dowling, T.I., Gallant, J.C. and Read, A.M. (2009), High Res DEMs for free18th IMACS World Congress MODSIM09, 13-17 Jul 2009, Cairns, Queensland, Australia., Conference CD.

Gallant, J. C., T. I. Dowling, A. M. Read, N. Wilson, P. K. Tickle, and C. Inskeep (2011). 1 second SRTMderived Digital Elevation Models User Guide. Geoscience Australia www.ga.gov.au/topographicmapping/digital-elevation-data.html.

Geoscience Australia (2008) Geodata 9 second DEM of Australia V3. Geoscience Australia, Canberra.

Hutchinson, M.F. (1989), A new method for gridding elevation and streamline data with automatic removal of pits. Journal of Hydrology 106:211-232.

Microsoft Corporation (2009), Photosynth, www.photosynth.net, (June 2009) 